\title{
Techniques tomographiques en écoulement diphasique
}

\author{
par Hervé Lemonnier, Jean-François Peytraud
}

du Laboratoire d'Etudes Fondamentales CEA, Grenoble

\section{INTRODUCTION}

La solution de certains problèmes industriels comme l'optimisation des réacteurs chimiques, la conception de mélangeurs ou de séparateurs, passe par la modélisation multidimensionnelle des écoulements diphasiques. L'étude et la modélisation de ces écoulements se heurtent, pour l'instant, au problème de la détermination pratique de la répartition spatiale des phases en présence. Pour faire progresser à la fois l'étude et la modélisation des écoulements diphasiques multidimensionnels, on a cherché à développer, au cours de cette étude, des techniques tomographiques en écoulement diphasique. Ces techniques ont pour objectif de permettre la détermination de la distribution du taux de présence dans un volume donné.

Les écoulements tridimensionnels ne présentant pas par définition de direction privilégiée, on s'intéresse a priori aux méthodes tomographiques en raison de leur absence de perturbation de l'écoulement. L'imagerie médicale a également recours à certaines techniques tomographiques comme le scanner $X$ ou la résonance magnétique nucléaire et l'imagerie d'impédance est à présent développée comme technique de substitution. Cette dernière technique, d'un coût d'investissement en principe plus léger, permettrait, d'une part, un premier diagnostique chez un médecin gé- néraliste et, d'autre part, de pratiquer l'imagerie fonctionnelle. Il s'agit ici d'accéder en temps réel à la déformation des organes internes en privilégiant la réponse dynamique à la résolution spatiale. L'objectif de notre étude est de déterminer dans quelle mesure cette technique est applicable aux écoulements diphasiques.

Le principe de la reconstruction tomographique sera brièvement rappelé et son mauvais conditionnement sera illustré par quelques exemples analytiques et numériques. Un algorithme de reconstruction original adapté aux écoulements diphasiques sera ensuite présenté. Cet algorithme bidimensionnel ne présente pas les défauts identifiés chez ses prédécesseurs. Puis, on en étudiera le conditionnement numérique et on évaluera l'influence des effets d'impédance interfaciale. Enfin on examinera les aptitudes de cet algorithme à reconstruire des données représentatives de capteurs réels obtenues par simulation tridimensionnelle.

\section{ERINCIPE DE LA TOMOGRAPHIE D'IMPÉDANCE}

La tomographie d'impédance opère en deux étapes. Le capteur procède d'abord à la caractérisation de la réponse

Impedance tomography consists in reconstructing the conductivity distribution from electrical data which characterise the electrical response of a medium to arbitrary excitations. Impedance tomography is an ill-conditioned problem and designing a tomograph therefore requires the quantitative knowledge of the sensitivity of the reconstruction to the measurements noise. The numerical conditioning of an original and accurate algorithm has been studied. This algorithm does not suffer from the shortcomings already identified in the literature. It is shown that for media encompassing inclusions which is a typical situation in two-phase flows, the necessary accuracy for the measurements if far beyond any technological reach. Moreover, within these high requirements for accuracy, some side effects must be carefully controlled or compensated and relevant procedures are provided. Furthermore, reconstruction artefacts are shown and they are found to derive from the unavoidable tridimensional nature of the electric field. For all these reasons, it is concluded that impedance tomography has very low potentialities as an accurate phase fraction distribution measuring technique in any arbitrary two-phase flows. 
du milieu à une sollicitation électrique arbitraire, puis reconstitue la distribution des propriétés électriques du milieu à partir de ces données. Les impédances mesurées en écoulement diphasique peuvent être de nature résistive comme par exemple dans l'étude de Jones et al. (1993) ou bien capacitives notamment lorsque les fluides sont isolants (par exemple Reinecke et Mewes, 1995).

La reconstruction tomographique est un problème inverse basé sur le problème direct du potentiel électrique dans un milieu de conductivité variable. Le problème capacitif est formellement analogue au problème résistif, la constante diélectrique jouant le rôle de la conductivité. Seul le problème résistif sera abordé ici, les résultats se transposant intégralement à la tomographie capacitive.

Dans un milieu $\Omega$ (fig. I) de conductivité variable, $\sigma(x)$, la distribution de potentiel obéit à la loi d'Ohm et la conservation du courant électrique. Supposons que le milieu soit excité en tension, le problème de Dirichlet qui en résulte s’écrit:

$$
\begin{gathered}
\nabla . \sigma \nabla V=0 \quad x \in \Omega \\
V(x)=V_{S}(s) \quad x \in \partial \Omega
\end{gathered}
$$

où $V$ est le potentiel électrique, $s$ est l'abscisse curviligne le long de $\partial \Omega$ et $V_{S}(s)$ est le potentiel d'excitation imposé sur la frontière du domaine. La résolution de (1) et (2) permet, outre le potentiel dans le milieu, de déterminer la densité de courant sur la frontière, qui d'après la loi d'Ohm s'écrit :

$$
j_{S}(s)=\left.\sigma \frac{\partial V}{\partial n}\right|_{x \in \partial \Omega}
$$

où $n$ est la normale extérieure au domaine.

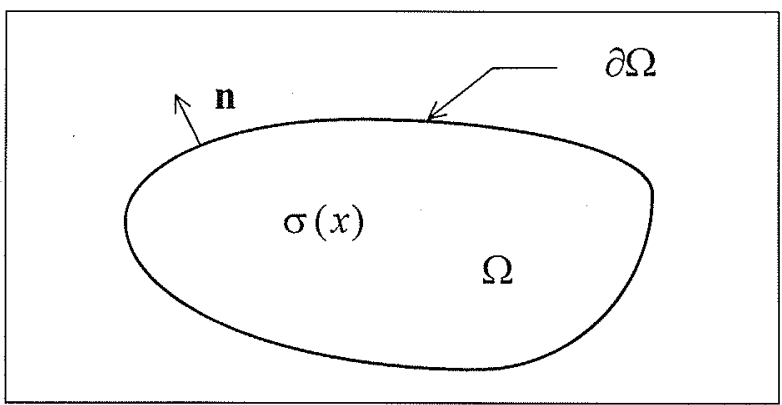

1. Description schématique du milieu électrique $(\Omega)$, de conductivité électrique $\sigma(x)$, pour la formulation du problème direct associé à la tomographie d'impédance résiptive.

A tout potentiel d'excitation arbitraire $V_{S}$ appliqué à la frontière du domaine, correspond une densité de courant $j_{S}$. Par la résolution de (1) et (2), on obtient $j_{S}$ connaissant $V_{S}$. Soit $\Lambda$ l'opérateur linéaire qui les relie:

$$
\Lambda: V(s) \stackrel{\Lambda}{\rightarrow}(\sigma) j(s) .
$$

L'opérateur $\Lambda$ caractérise entièrement le comportement électrique du milieu. Si le milieu porte des électrodes (fig. 2), le problème direct est alors un problème mixte : le potentiel est constant et imposé sur les électrodes tandis que la densité de courant est nulle entre chaque électrode. On montre (Peytraud, 1995) que pour un ensemble de tensions d'excitation $V$ la solution de (1) donne les courants transitant par les électrodes $l$. Le problème mixte est alors caractérisé par la matrice d'admittance $Y(\sigma)$ du capteur :

$$
V\left(V_{1}, V_{2}, V_{3}, \ldots, V_{n}\right) \stackrel{Y(\sigma)}{\rightarrow} I\left(I_{1}, I_{2}, I_{3}, \ldots, I_{n}\right)
$$

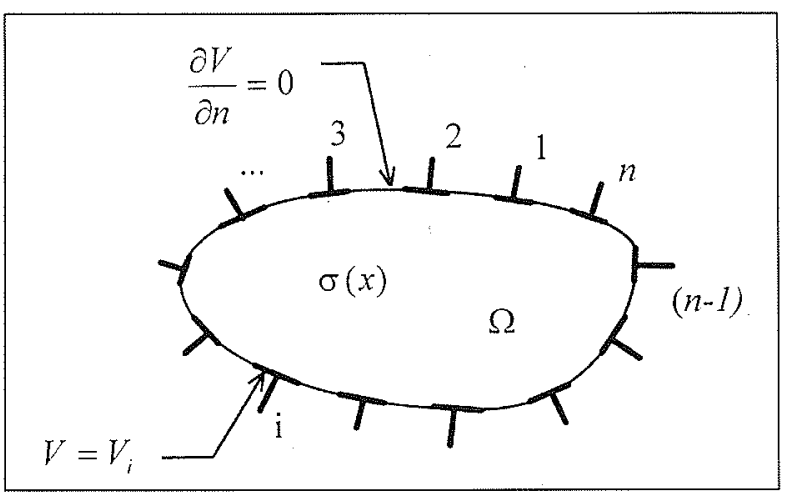

2. Description schématique d'un milieu portant $n$ électrodes d'excitation (en gras) et de mesure.

La tomographie d'impédance consiste simplement à retrouver $\sigma$ lorsque $\Lambda$ ou plus pratiquement $Y$ sont connus. De plus, $\Lambda$ comme $Y$ sont symétriques. Il résulte que la matrice d'admittance d'un système comportant $n$ électrodes ne comprend que $n(n-1) / 2$ coefficients indépendants. Cette dernière quantité représente aussi le nombre maximum de paramètres caractérisant le milieu qui peuvent être déterminés sans sous-détermination.

\section{CONDITIONNEMENT DU PROBLÈME INVERSE}

Andersen et Bernsten (1988) ont montré que le problème direct (1) et (2) pour un milieu circulaire avait une solution analytique par séparation des variables lorsque la distribution de conductivité présente une symétrie de révolution $(\sigma(r))$. Le problème inverse linéarisé possède également une solution analytique pour des distributions de conductivité polynomiales (Andersen et Bernsten, 1988) ou encore une solution numérique exacte pour des répartitions de conductivité constantes par morceau (Seagar et Bates, 1985).

Andersen et Bernsten (1988) montrent que l'inversion tomographique ne permet pas de déterminer la conductivité au centre du cylindre et d'après eux, le mauvais conditionnement en résulte. Leur solution est obtenue sous la forme d'un développement selon des polynômes de Legendre et la sensibilité au bruit de la détermination des coefficients augmente exponentiellement avec le nombre de termes du développement.

La figure 3 montre la reconstruction d'un milieu initial formé seulement de 15 anneaux de conductivité uniforme et de surface égale (b). Les données de reconstruction 

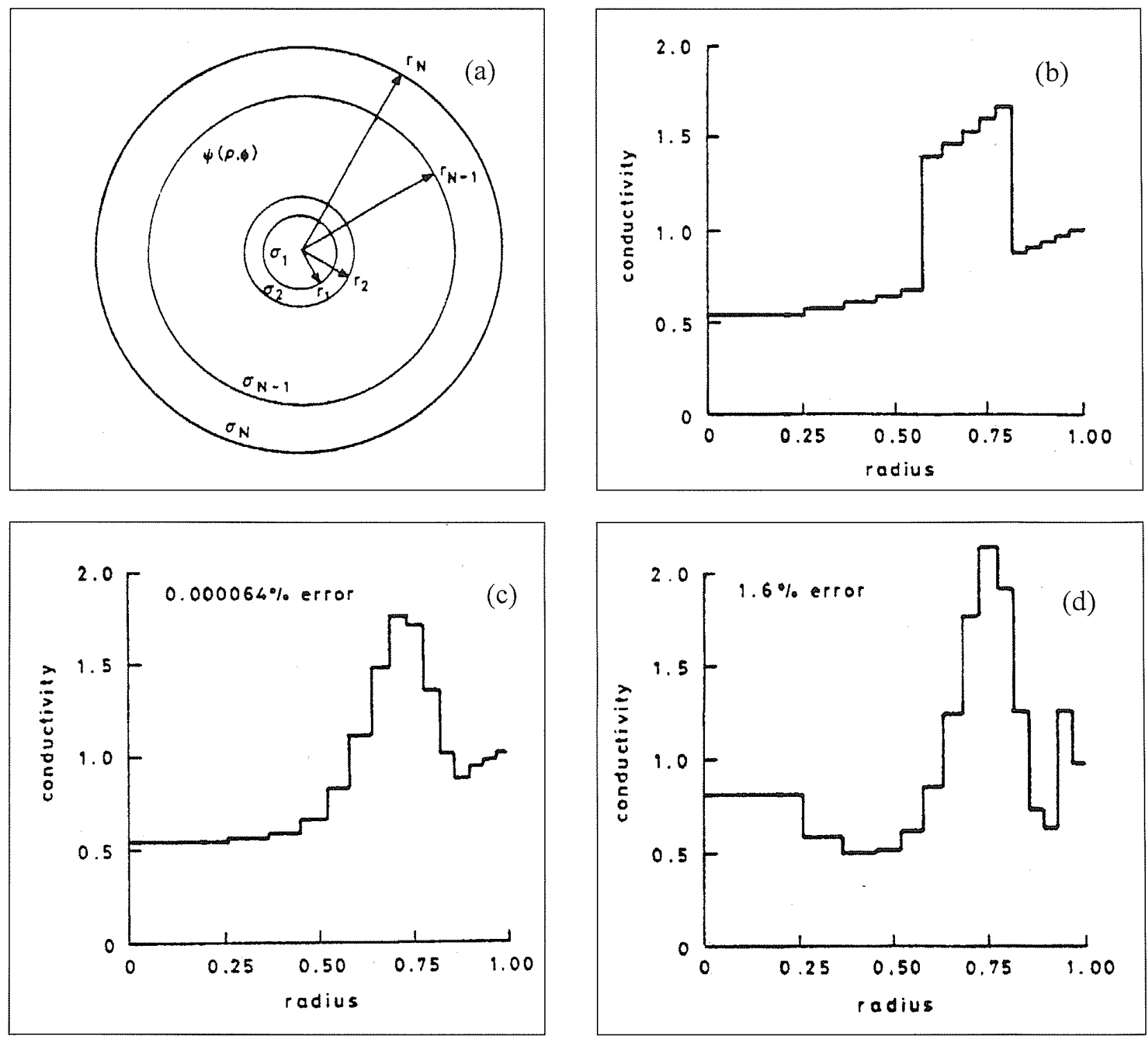

3. Reconstructions de profils de conductivité pour un milieu circulaire axisymétrique selon Seagar et Bates (1985). L'original $(b)$ est reconstruit imparfaitement même lorsque les données sont entachées d'un bruit minime $(c)$. La reconstruction est erronée lorsque le bruit est important $(d)$.

tomographique sont simulées en résolvant le problème direct puis sont ensuite entachées de bruit. La figure $3 c$ montre que même lorsque ce bruit est extrêmement faible $\left(<10^{-6}\right)$, la reconstruction est significativement erronée mais reste toutefois acceptable à la périphérie. En revanche, lorsque le bruit augmente $\left(\sim 10^{-2}\right)$, la reconstruction est de très mauvaise qualité. Ces résultats étonnants sont néanmoins (et malheureusement) faciles à reproduire et illustrent la sensibilité extrême au bruit de la reconstruction tomographique.

Bernsten et al. (1991) ont étendu leur solution analytique linéarisée au cas d'un cylindre de conductivité quelconque. La conductivité inconnue est recherchée sous la forme du développement suivant:

$$
\sigma=\sum_{n=0}^{n=N} \sigma_{n}(r) e^{i m \theta} \quad \text { où } \quad \sigma_{n}(r)=\sum_{m=0}^{n=M} A_{m n} P_{m}(r)
$$

où $N$ est le nombre d'harmoniques azimutales et $M$, le degré de la partie radiale. La figure $4 a$ montre la distribution à retrouver. Elle est typique des écoulements diphasiques car elle présente une discontinuité de conductivité. On observe (fig. $3 b$ à $d$ ) que plus le nombre de termes de (6) augmente, plus la reconstruction se dégrade et plus l'amplitude des oscillations de la reconstruction augmente.

Seagar et Bates (1985), à l'aide d'une technique analytique, mettent en évidence ce que, pour des milieux à inclusions, on pourrait appeler une ambiguitté tailleconductivité. En effet, la précision nécessaire pour déterminer précisément à la fois la taille et la conductivité d'une inclusion est bien plus grande que pour déterminer l'une connaissant l'autre.

Ces résultats sont confirmés par une étude numérique de Yorkey (1986) qui a résolu le problème inverse par la 

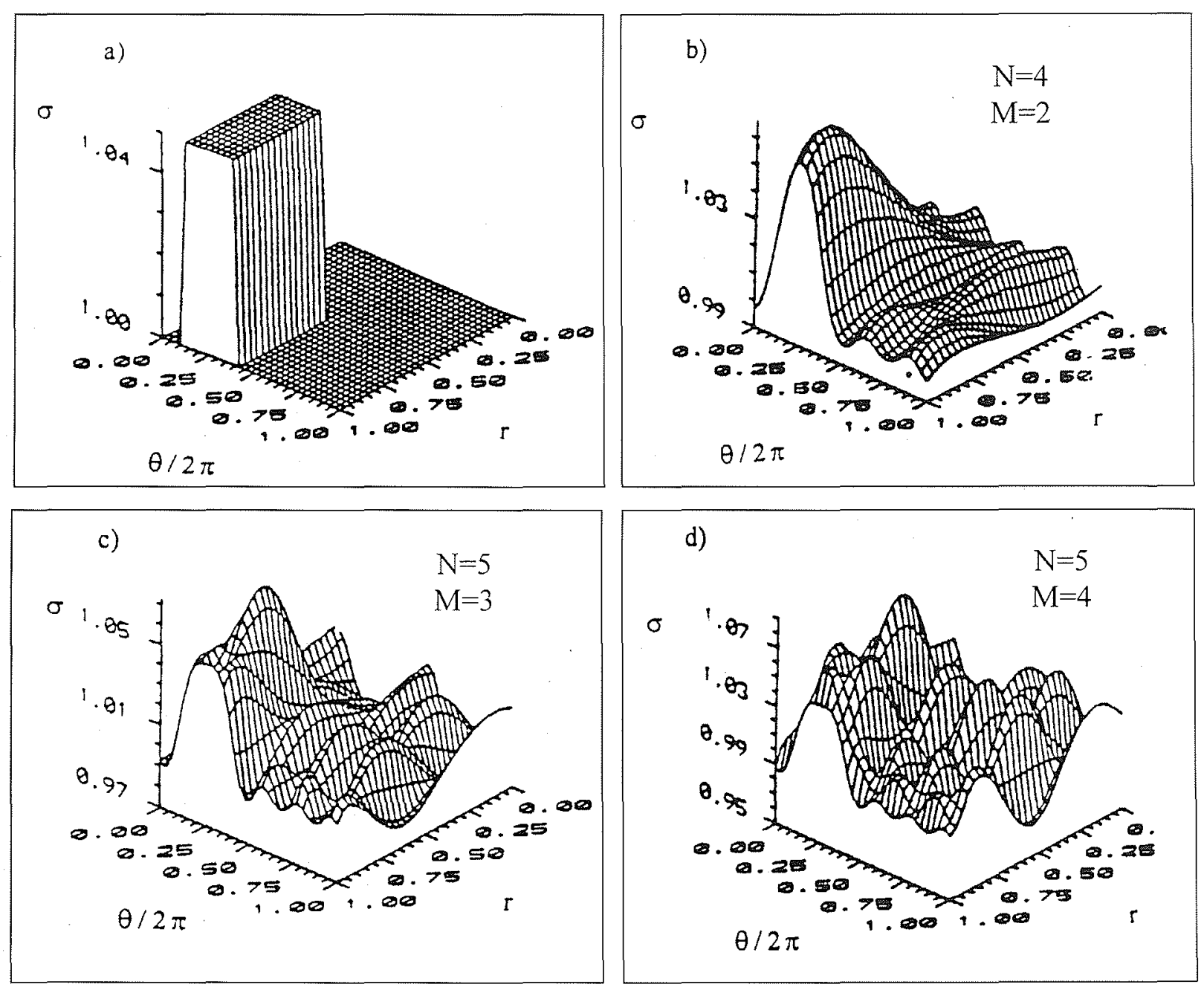

4. Reconstructions de distribution de conductivité $(\sigma)$ bidimensionnelle, par la méthode de Bernsten et al. (1991). $L$ 'original $a$ ) voit sa reconstruction se dégrader lorsque l'on augmente le nombre de terme de la série de reconstruction (6). Les oscillations de la solution croissent de $b$ ) à $d$ ).

méthode des éléments finis. La sensibilité au bruit a été également identifiée et, à l'aide d'une décomposition en valeurs singulières, Yorkey montre que les éléments les plus sensibles sont placés près de la périphérie du domaine tandis que ceux du centre sont affectés d'une sensibilité très faible, le rapport entre ces sensibilités extrêmes pouvant atteindre $10^{-6}$ ! De plus les méthodes d'éléments'finis ont des performances discutables (Jones et al., 1993) pour des milieux à conductivité constante par morceau et éprouvent des difficultés à reproduire les discontinuités qui n'épousent pas parfaitement le maillage ( $f$ ig. 5).

A l'issue de l'analyse de la bibliographie, Peytraud (1995) recommande d'étudier le conditionnement de l'algorithme d'inversion afin de pouvoir déterminer la précision avec laquelle les données de reconstruction devront être obtenues. C'est un point crucial pour la conception d'un tomographe à impédance pour les écoulements diphasiques. En effet, la nature dynamique des écoulements nécessite d'acquérir les données en un temps court afin d'éviter les effets de flou. La précision entrant nécessairement en conflit avec la rapidité, le meilleur compromis rapidité - réponse dynamique - résolution ne peut être obtenu sans caractériser l'algorithme.
Peytraud (1995), au vu du mauvais conditionnement et du nombre limité d'informations que l'on peut extraire du milieu, recommande de prendre en compte toutes les informations connues, ce qui le conduit à recommander l'usage de la méthode des éléments de frontière pour l'algorithme de reconstruction. Cela permet d'éviter les inconvénients des algorithmes existants.

\section{ALGORITHME DE RECONSTRUC- TION BIDIMENSIONNEL}

Le milieu d'étude sera dorénavant considéré comme constitué de deux phases dont les propriétés électriques seront supposées distinctes, constantes et uniformes. On recherchera la solution au problème de reconstruction sous la forme d'un ensemble d'inclusions de conductivité $\sigma_{k}$ plongées dans un milieu de conductivité $\sigma$ (fig. 6).

Dans ces conditions, le potentiel est harmonique dans chacune des phases et, à chaque interface, le potentiel et la densité de courant sont continus.

La méthode des éléments de frontière prend en compte parfaitement le caractère discontinu de la distribution de 

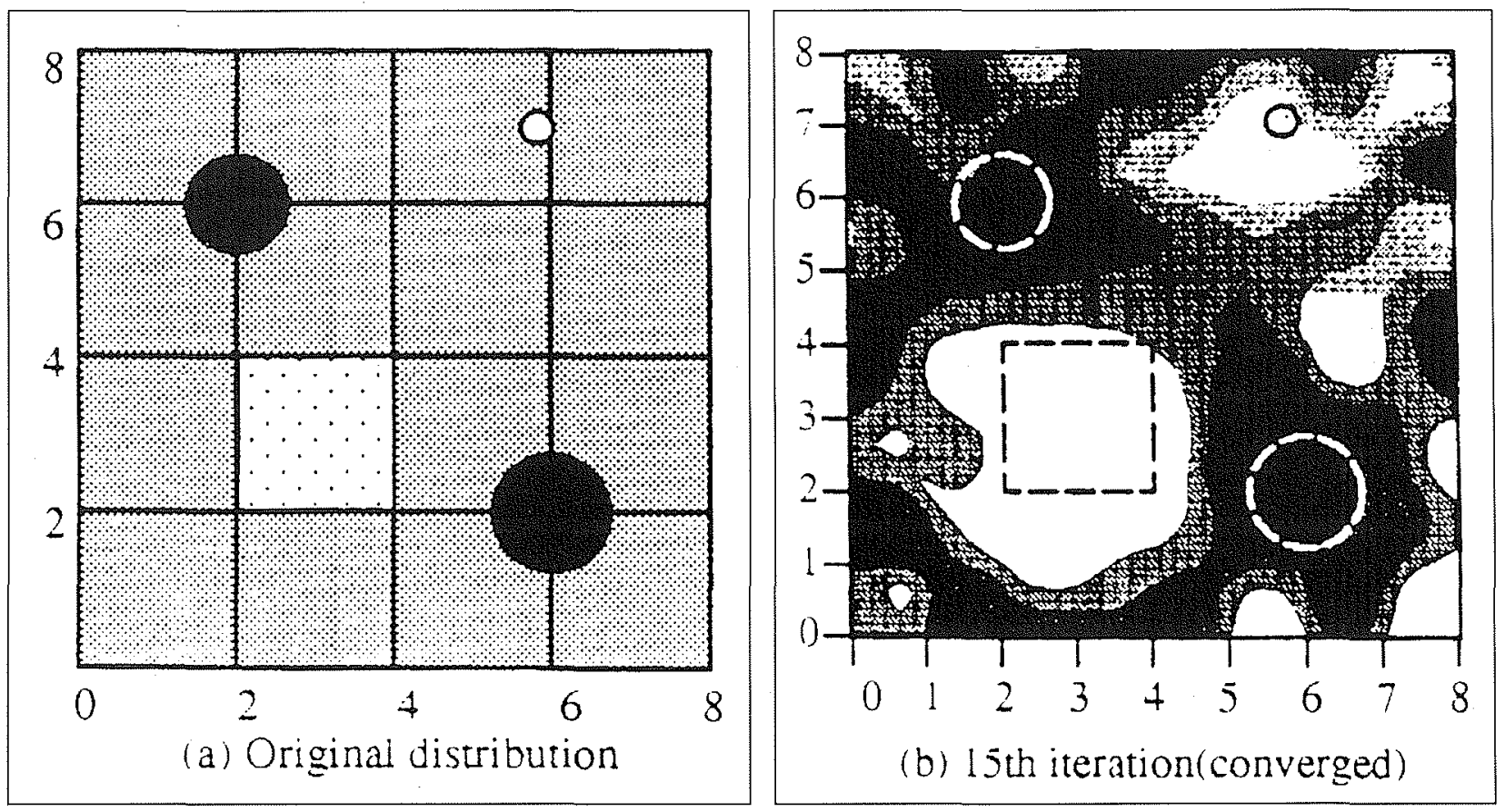

5. Exemple de reconstruction par éléments finis d'un milieu à inclusions de conductivité constante. D'après Jones et al. $\cdot(1993)$.

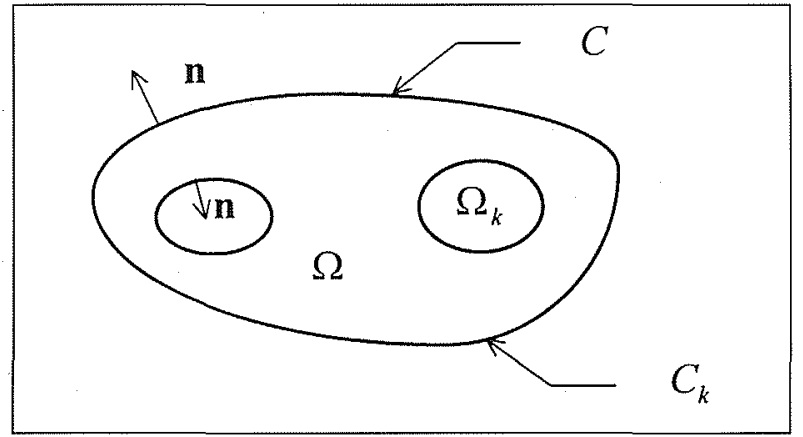

6. Définitions relatives à la formulation du problème direct de la tomographie par la méthode des équations intégrales.

conductivité et permet de résoudre avec précision le problème direct. Elle est basée sur la résolution de l'équation de Laplace par la méthode des équations intégrales dont une introduction superficielle est fournie par Brebbia et Dominguez (1992), de nombreux problèmes numériques trouvant leur solution pratique dans la monographie de Hess et Smith (1967).

\subsection{Méthode intégrale pour l'équation de Laplace}

Le principe de la méthode intégrale se présente facilement à propos du problème de Dirichlet intérieur. Son extension aux problèmes de Neumann et mixte est immédiate (Brebbia et Dominguez, 1992). Soit le problème de Dirichlet suivant pour $V$, le potentiel électrique :

$$
\begin{array}{r}
\nabla^{2} V=0 \quad x \in \Omega \\
V=V_{S}(x) \quad x \in \partial \Omega
\end{array}
$$

La résolution du problème est simplifiée si l'on connaît la fonction de Green du problème. Généralement on utilise la fonction de Green du problème de Dirichlet homogène à l'infini dont les propriétés caractéristiques sont :

$$
\begin{aligned}
& \nabla^{2} G=\delta\left(x-x^{\prime}\right) \\
& \lim _{x \rightarrow \infty} G\left(x, x^{\prime}\right)=0
\end{aligned}
$$

$G$ représente le potentiel engendré en $x$ par une source placée en $x^{\prime}$. Cette fonction est connue en 2D comme en 3D. L'utilisation du théorème de Green et l'analyse de la continuité des différentes intégrales permettent ensuite d'obtenir les relations suivantes:

$$
\left.\begin{array}{cc}
V(x) & x \in \Omega \\
1 / 2 V(x) & x \in \delta \Omega \\
0 & x \notin(\Omega \cup \delta \Omega)
\end{array}\right\}=\int_{\delta \Omega}\left(V \frac{\partial G}{\partial n}-G \frac{\partial V}{\partial n}\right) \mathrm{d} S^{\prime}
$$

La première ligne de (9) signifie que le potentiel dans le domaine ne dépend que de la valeur de deux fonctions, $V(s)$ et $\partial V / d n$ sur le contour du domaine. Cette identité illustre la propriété fondamentale des méthodes des équations intégrales : la réduction de la dimension des problèmes d'une unité. La deuxième ligne exprime la relation intégrale qui existe entre le potentiel. et la densité de courant. Dans le cas du problème de Dirichlet la dérivée normale du potentiel, $J$, est solution d'une équation de Fredholm de première espèce

$\int_{\delta \Omega} J\left(x^{\prime}\right) G\left(x, x^{\prime}\right) \mathrm{d} S^{\prime}=\int_{\delta \Omega} V_{S}\left(x^{\prime}\right) \frac{\partial G}{\partial n}\left(x, x^{\prime}\right) \mathrm{d} S^{\prime}-\frac{1}{2} V_{s}(x)$ 
Cette expression exprime la relation intégyale et explicite entre le potentiel et la densité de courant sur la frontière et représente très précisément ce que l'on recherchait, démontrant ainsi l'adéquation parfaite de la méthode des intégrales de frontière à la résolution du problème direct de la tomographie d'impédance.

\subsection{Méthode des éléments de frontière pour la résolution du problème direct}

Le problème direct de la tomographique d'impédance consiste à calculer, pour un milieu arbitraire, les courants d'électrode résultant d'applications de potentiels (5). Le domaine, décrit à la figure 6 , consiste en un milieu continu de conductivité constante $\sigma$ dans lequel sont plongées des inclusions de conductivité constante $\sigma_{k}$. On obtient la formulation du problème direct en écrivant l'identité de Green (9) pour le domaine continu $\Omega$, limité par $C$ et la frontière des inclusions $C_{k}$, et chaque inclusion $\Omega_{k}$. On combine les identités obtenues en les multipliant par la conductivité du milieu correspondant et en les ajoutant. On fait alors apparaître sur $C_{k}$ la différence des densités de courant de part et d'autre de la frontière. En tenant compte de la continuité de la densité de courant et du potentiel à la frontière des inclusions on obtient :

$$
\begin{aligned}
& \left.\begin{array}{cc}
1 / 2 \sigma V & x \in C \\
\sigma V & x \in \Omega
\end{array}\right\} \quad \sigma \int_{C}\left(V \frac{\partial G}{\partial n}-G \frac{\partial V}{\partial n}\right) \mathrm{d} S \\
& \left.\frac{\sigma_{k}+\sigma}{2} V x \in C_{k}\right\}=+\sum_{k}\left(\sigma-\sigma_{k}\right) \int_{C_{k}} V \frac{\partial G}{\partial n} \mathrm{~d} S
\end{aligned}
$$

Seagar et Bates (1985), ont résolu cette équation intégrale en développant le potentiel et la densité de courant sur une base de fonctions appropriée. L'équation intégrale (11) fut alors projetée sur la même base, ce qui produit un système algébrique linéaire infini. La résolution de ce système, après troncature, permet d'approcher la solution. Malheureusement, la nature mixte de la frontière $C$, constituée alternativement d'électrodes conductrices et de sections isolantes, rend la méthode peu convergente. En effet, en absence d'inclusion, la méthode de Seagar et Bates est identique à la méthode de séparation des variables. La solution analytique du problème est alors disponible pour certaines configurations d'électrodes et, de plus, son comportement local est connu : le potentiel et la fonction de courant sont finis mais de variations non bornées à la limite électrode-paroi isolante. Cette singularité impose au terme général de la série représentant de la solution de décroître très lentement en module (comme $1 / n$ ). Cette situation est très défavorable numériquement et nous a incités définitivement à préférer la méthode des éléments de frontière.

La méthode des éléments de frontière (MEF) consiste à résoudre (11) par une méthode de collocation. Cette équation est écrite pour un certain nombre de points de collocation, $x_{i}$, placés sur les frontières $C$ et $C_{k}$. Puis les intégrales sont évaluées en approchant les frontières par une ligne polygonale et en considérant que les fonctions inconnues, $V$ et $j$ sont constantes sur chaque élément de la frontière. Il convient de choisir autant d'éléments que de points de collocation et on place le point de collocation au centre de chaque élément $\Delta$. Les valeurs discrètes du potentiel, $V_{i}$, et de la densité de courant, $j_{i}$, sont alors linéairement liées par:

$$
\left\{\begin{array}{cc}
1 / 2 \sigma V_{i} & x_{i} \in C \\
1 / 2 & \left(\sigma_{k}+\sigma\right) V_{i} x_{i} \in C_{k}
\end{array}\right\}=+\begin{gathered}
\sigma \sum_{x, \in} H_{i j} V_{j}-G_{i j} J_{j} \\
\sum_{k}\left(\sigma-\sigma_{k}\right) \sum_{x_{i} \in C_{k}} H_{i j} V_{j}
\end{gathered}
$$

où $H_{i j}$ et $G_{i j}$ sont des constantes ne dépendant que du choix des éléments et des points de collocation (maillage). Ces constantes s'expriment par des intégrales qui sont calculables par des fonctions élémentaires (Hess et Smith, 1967 Peytraud, 1995) pour des éléments rectilignes (2D) et des éléments à contour polygonal (3D) :

$$
H_{i j} \triangleq \int_{\Delta_{j}} \frac{\partial G}{\partial n}\left(x_{i}, x\right) \mathrm{d} x \quad G_{i j} \triangleq \int_{\Delta_{i}} G\left(x_{i}, x\right) \mathrm{d} x
$$

L'équation 12 représente la relation algébrique liant les valeurs de $V$ et $j$ sur la frontière portant les électrodes et les valeurs de $V$ sur la frontière des inclusions. Le potentiel est connu sur les électrodes tandis que la densité de courant est connue (nulle) sur les sections isolantes séparant les électrodes. On peut alors obtenir par résolution de (12) les valeurs manquantes : le potentiel sur les inclusions et les sections isolantes de la frontière extérieure et surtout la densité de courant sur les électrodes.

Avec quelques centaines d'éléments on obtient une très bonne précision. La comparaison de la MEF avec des solutions analytiques de problèmes mixtes (Peytraud. 1995) montre que pour le problème mixte à quatre électrodes et un milieu homogène, le courant traversant le milieu est calculé à mieux que $10^{-4}$ près avec seulement 400 éléments. Des éléments d'ordre plus élevé existent (Brebbia et Dominguez, 1992) pour résoudre (11), mais les bons résultats obtenus avec les éléments linéaires ne justifient pas cet accroissement de complexité.

\subsection{Problème inverse : algorithme de reconstruction}

La simulation du problème direct par la MEF permet d'obtenir la matrice d'admittance du milieu $\boldsymbol{Y}$. Chaque inclusion du milieu est caractérisée par son centre $\left(x_{k}, y_{k}\right)$ et son rayon $a_{k}$. Ces paramètres caractérisent le milieu et seront nommés collectivement $q_{k}$. Le problème de la reconstruction est un problème d'optimisation où la fonction objectif peut être définie par:

$$
E^{2}\left(q_{k}\right)=\sum_{i}\left(Y_{i}\left(q_{k}\right)-Y_{i}^{00}\right)^{2}
$$

Il s'agit de trouver les $q_{k}$ qui minimisent $E$. On utilise la méthode de Newton-Raphson pour résoudre ce problème non linéaire. A partir d'une estimation initiale du milieu $q_{k}^{0}$, même prise au hasard, les mesures sur ce milieu permettent de déterminer la matrice d'admittance $Y^{00}$. On montre que le minimum de $E$ peut être approché en résolvant le système linéaire suivant :

$$
\sum_{i} \frac{\partial Y_{i}}{\partial q_{k}} \frac{\partial Y_{i}}{\partial q_{l}} \Delta q_{k}=\sum_{i} \frac{\partial Y_{l}}{\partial q_{i}}\left(Y_{l}^{00}-Y_{l}^{0}\right)
$$

où $\Delta q_{k}$ sont les corrections à appliquer aux paramètres de structure $q_{k}$ et $Y^{0}$, est la matrice d'admittance correspondant à l'estimation initiale $q_{k}^{0}$. La sensibilité au bruit de l'algorithme de reconstruction peut être analysée en étudiant le nombre de conditionnement de l'opérateur figurant au membre de gauche de (15). 
La résolution de (15) nécessite le calcul de la sensibilité de la solution du problème direct aux variations des paramètres de structure. Une estimation simple peut être obtenue par différences finies en effectuant deux calculs directs et en perturbant un des $q_{k}$ pour le second. Cette méthode est lourde, $3 N+1$ calculs directs à chaque itération de Newton-Raphson. On propose une méthode de perturbation qui ne nécessite que très peu d'efforts supplémentaires à la suite d'un seul calcul direct.

\subsection{Calcul des sensibilités par une méthode de pertur- bation régulière}

Pour calculer la sensibilité de la solution du problème direct aux variations de l'inclusion $k$, on considère le problème direct posé sur un domaine perturbé où l'inclusion $k$, initialement circulaire a été déformée en une nouvelle surface, $\Sigma_{\infty}$, dont l'équation polaire est :

$$
\Sigma_{\varepsilon}: r=a_{k}+\varepsilon f(s)
$$

où $s$ est labscisse curviligne le long de la courbe non déformée. On cherche ensuite à déterminer la solution sous la forme :

$$
V=V_{0}+\varepsilon V_{1}+O\left(\varepsilon^{2}\right)
$$

On a évidemment :

$$
V_{1}=\left.\frac{\partial V}{\partial \varepsilon}\right|_{\varepsilon=0}
$$

II s'avère que $V_{1}$ est la solution d'un problème analogue à $V_{0}$, problème non perturbé déjà résolu. En effet, le problème d'ordre 1 est posé sur la même géométrie que celle du problème d'ordre 0 . De plus les conditions aux limites sont de même type pour les deux problèmes. Pour le problème d'ordre 1 , elles sont homogènes partout sauf sur l'inclusion $k$ où la condition de courant nul est remplacée par:

$$
\frac{\partial V_{1}}{\partial n}=\frac{\partial}{\partial s} f \frac{\partial V_{0}}{\partial s}
$$

où $s$ est l'abscisse curviligne le long du contour de l'inclusion. (19) généralise le résultat présenté par Lemonnier et Peytraud (1995) pour des inclusions circulaires. En choisissant $f$ des façon appropriée on obtient les sensibilite aux différents paramètres de structure. Ce choix est le suivant :

$$
\begin{gathered}
f=1 \rightarrow V_{1}=\frac{\partial V}{\partial a_{k}} \\
f=\cos (\theta) \rightarrow V_{1}=\frac{\partial V}{\partial x_{k}} \\
f=\sin (\theta) \rightarrow V_{1}=\frac{\partial V}{\partial y_{k}}
\end{gathered}
$$

Le problème d'ordre 0 produit un système linéaire que l'on résout simplement par la technique de décomposition triangulaire de Gauss. Il est remarquable de constater que le problème d'ordre 1 comporte le même premier membre que le problème d'ordre 0 est qu'il est donc par conséquent déjà inversé. Le calcul de la sensibilité se limite ainsi au calcul du second membre de (19) et à l'évaluation de la solution. En aucun cas on ne recalcule les coefficients d'influence $G$ ou $H$ ni la solution de (12) comme cela aurait été le cas pour la méthode par différences finies.

\subsection{Mise en ceuvre pratique de l'algorithme}

Le nombre d'inclusions n'est pas connu au moment du calcul. La technique qui s'est avérée la plus fiable consiste à démarrer le calcul inverse avec le nombre maximum d'inclusions compatible avec le nombre de données indépendantes. Sachant que chaque inclusion est représentée par 3 paramètres, ce nombre maximum d'inclusions, $l$, vérifie :

$$
3 l \leqslant n(n-1) / 2
$$

Les inclusions de départ pour la reconstruction sont choisies petites $(0,5$ fois le rayon du capteur) et sont disposées au hasard. Lorsqu'au cours des itérations une inclusion voit son rayon diminuer constamment, elle est simplement éliminée. Pour tous les calculs présentés, les itérations sont arrêtées lorsque la norme des corrections est inférieure à $10^{-6}$

\section{EFFET DU BRUIT SUR LA RECONS- TRUCTION}

La figure 7 montre 4 reconstructions de milieux à 6 inclusions dans un capteur tomographique portant 8 électrodes et dont la longueur cumulée représente la moitié du périmètre disponible. Les données de reconstruction ont tout d'abord été simulées par un calcul direct puis, la reconstruction a été effectuée par l'algorithme décrit au paragraphe précédent.

Peytraud (1995) a utilisé l'algorithme de Marquadt (1963) qui permet de forcer la convergence vers un minimum (local) de la fonction objectif. Même sans utiliser cette procédure, l'algorithme de Newton-Raphson converge néanmoins assez souvent vers le minimum absolu de la fonction objectif, comme le montre la figure 7 : pour 4 tirages aléatoires de la distribution de départ, deux calculs ont convergé vers la solution. On remarque que le minimum atteint par la fonction objectif est 0 lorsqu'il y a convergence vers la solution et de l'ordre de $10^{-2}$ lorsque la convergence a lieu vers un minimum local de $E$ (14). La valeur minimum de la fonction objectif après convergence du calcul est donc le seul critère objectif de vraisemblance de la solution.

La reconstruction à jusqu'à présent été effectuée sur des données $(Y)$ simulées par un calcul direct. On effectuera la reconstruction sur des données bruitées:

$$
Y_{i j}^{\prime}=Y_{i j}+\varepsilon x(\omega)
$$

où $\varepsilon$ est le niveau de bruit et $x(\omega)$ est une variable aléatoire comprise entre -1 et 1 de densité de probabilité constante. La figure 8 montre que la qualité de la reconstruction se dégrade légèrement avec l'augmentation du niveau de bruit. Le nombre d'inclusions est dans ce cas. bien choisi, correctement estimé et la reconstruction s'avère qualitativement correcte. On remarque cependant que la fonction objectif tend, à la convergence, vers une valeur proportionnelle au niveau de bruit. Même pour la valeur la plus faible valeur du bruit $\left(10^{-3}\right.$, figure $\left.8 d\right)$, la 
reconstruction est correcte tandis que la fonction objectif atteint des valeurs analogues à celles atteinte à la figure 7 ( $c$ et $d$ ). Il est clair que le bruit n'empêche pas la reconstruction de se dérouler normalement ce qui prouve la solidité de notre algorithme. Cependant, l'existence de bruit sur les données rend inutilisable le critère de vraisemblance de la solution qui permet de discriminer une mauvaise solution (figs. $7 c$ et $7 d$ ) d'une reconstruction correcte mais entachée de bruit (figs $8 b, 8 c, 8 d$ ).

\section{VI 圆 QUANTIFICATION DU CONDITION- NEMENT NUMÉRIQUE DE L'ALGO- RITHME}

Le conditionnement de l'algorithme est précisément lié au nombre de conditionnement de $U$ l'opérateur linéaire de (15).
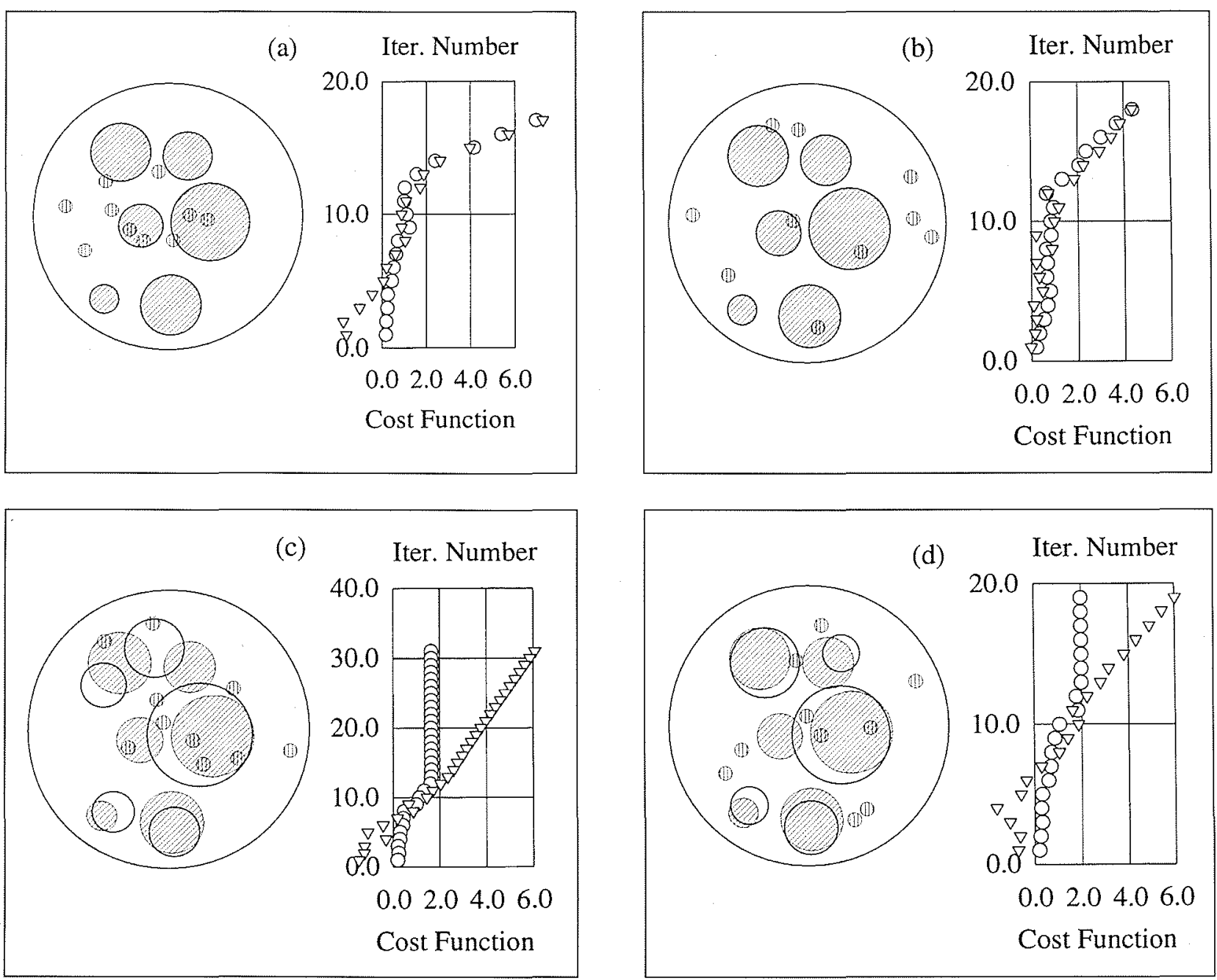

7. Reconstructions à partir de données simulées (8 électrodes équidistantes). Les contours en traits pleins représentent la reconstruction, les cercles rayés verticalement représentent la configuration initiale et les cercles portant les rayures obliques représentent le milieu qu'il fallait effectivement retrouver. Le graphique de droite de chaque figure représente l'opposé du logarithme décimal de la fonction coût et du module des corrections en fonction du numéro de l'itération. Dans les 4 cas l'algorithme converge, le module des itérations $(\nabla)$ tendant vers zéro. Seulement dans les deux figures du haut, l'algorithme converge vers le minimum absolu de la fonction objectif $(O)$ qui tend alors vers zéro. 

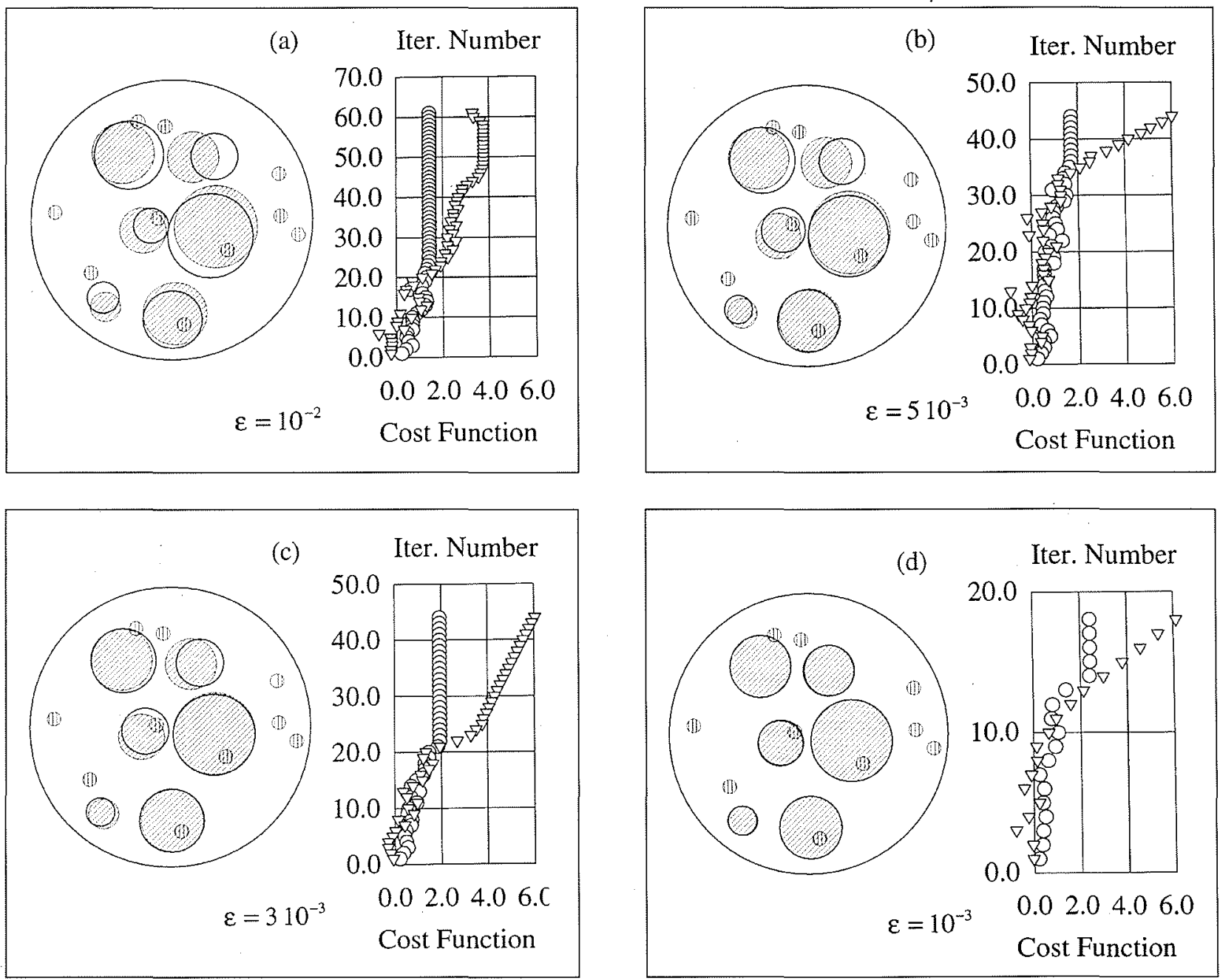

8. Reconstructions à partir de données simulées et entachées de bruit. Voir la légende de la figure 7 pour les définitions. Le niveau de bruit diminue depuis $(a)$ jusqu'à $(d)$. On remarque que la reconstruction est acceptable voire bonne mais que la fonction objectif $(O)$ ne tend pas vers zéro mais vers une valeur proportionnelle au niveau de bruit $(\varepsilon)$.

Tableau 1. - Valeurs moyennes du nombre de conditionnement $k(U)$ et de la précision de mesure nécessaires $\left(\eta_{v}\right)$ à la restitution des paramètres descriptif du milieu $q_{k}$ à $1 \%$ près en fonction du nombre d'inclusions.

\begin{tabular}{|c|c|c|c|c|c|c|}
\hline Nbre d'Inclusions. & 1 & 2 & 3 & 4 & 5 & 8 \\
\hline$k(U)$ & $10^{2}$ & $10^{2}$ & $10^{3}$ & $10^{4}$ & $10^{5}$ & $10^{6}$ \\
\hline$\eta_{r}$ & $10^{-4}$ & $10^{-5}$ & $10^{-6}$ & $10^{-8}$ & $10^{-9}$ & $10^{-11}$ \\
\hline
\end{tabular}

Par simulation numérique, il a été possible de déterminer des valeurs moyennes du nombre de conditionnement en fonction du nombre d'inclusions à reconstruire. Peytraud, (1995) en déduit la précision sur les mesures de tension et de courant afin de reconstruire le milieu avec une précision donnée. Cette précision est directement proportionnelle à l'incertitude que l'on accepte sur la détermination de la position et du rayon des inclusions. Le tableau 1 donne les précisions relatives à la détermination à $1 \%$ de la géométrie des inclusions. On observe que pour 3 inclusions une valeur moyenne de la précision nécessaire est de $10^{-6}$, ce qui est typique de la précision de ponts de mesure ne fonctionnant qu'en statique. Autant dire que reconstruire plus de quatre inclusions en situation d'écoulement est en deçà de toutes possibilités technologiques.

\section{EFFET DES IMPÉDANCES D'INTER- FACE}

L'impédance d'interface est un phénomène prenant place au voisinage immédiat de l'interface électrode-milieu. II se manifeste par le fait que le potentiel effectivement appliqué au milieu est différent du potentiel de l'électrode. Ce phénomène résulte d'un certain nombre de mécanismes comme les réactions de transfert de charges dont la cinétique n'est pas instantanée el qui nécessitent une énergie d'activation finie. Ces réactions peuvent être ralenties par la diffusion des espèces dans le milieu ou bien modifiées 
par des réactions secondaires en surface ou dans le volume de l'électrolyte. De plus, l'impédance d'interface est sensible à l'état de surface.

Cette impédance pour le couple eau-inox dépend de la fréquence $\left(\infty f^{-1 / 2}\right)$ et possède une partie réelle et imaginaire de même ordre de grandeur. Cette impédance se place en série avec celle de la cellule de mesure et Peytraud (1995) a caractérisé ce phénomène sur un prototype de capteur plan. Il montre que ce phénomène est significatif dans la bande de fréquence allant de $100 \mathrm{~Hz}$ à $10 \mathrm{kHz}$ qui est typiquement celle utilisée pour les capteurs à impédance. Pour le couple eau-inox, à $100 \mathrm{~Hz}$, l'impédance d'interface représente typiquement $5 \%$ de l'impédance totale mesurée.

On peut tenir compte de cet effet dans la MEF. La condition de.Dirichlet sur l'électrode est remplacée par une condition mixte:

$$
V+z \frac{\partial V}{\partial n}=V_{s}(x)
$$

où z est l'impédance d'interface saras dimension (Peytraud, 1995) et $V_{s}$ est le potentiel d'électrode. L'impédance sans dimension représente le rapport des effets d'interface aux effets purement résistifs dans le milieu. Lorsque $z$ est nul, l'interface est parfaite.

Pour les besoins de la démonstration, des données ont été simulées en prenant en compte l'impédance d'interface (z) tandis que des reconstructions ont été effectuées en considérant que l'interface était parfaite. Quelques résultats sont indiqués à la figure 9 où on montre que l'effet global de l'impédance d'interface est comparable à celui du bruit.

Pour les faibles valeurs de l'impédance sans dimension (z) la reconstruction est correctement effectuée mais le minimum de la fonction objectif n'est pas nul. Comme dans le cas de la reconstruction de données bruitées, ce minimum est proportionnel à z. Lorsque les effets d'impédance sont plus marqués, on observe que la reconstruction se dégrade.

Cependant contrairement aux effets du bruit qui sont aléatoires et que l'algorithme peut éliminer, les effets d’impédance d’interface sont systématiques. En effet, négliger
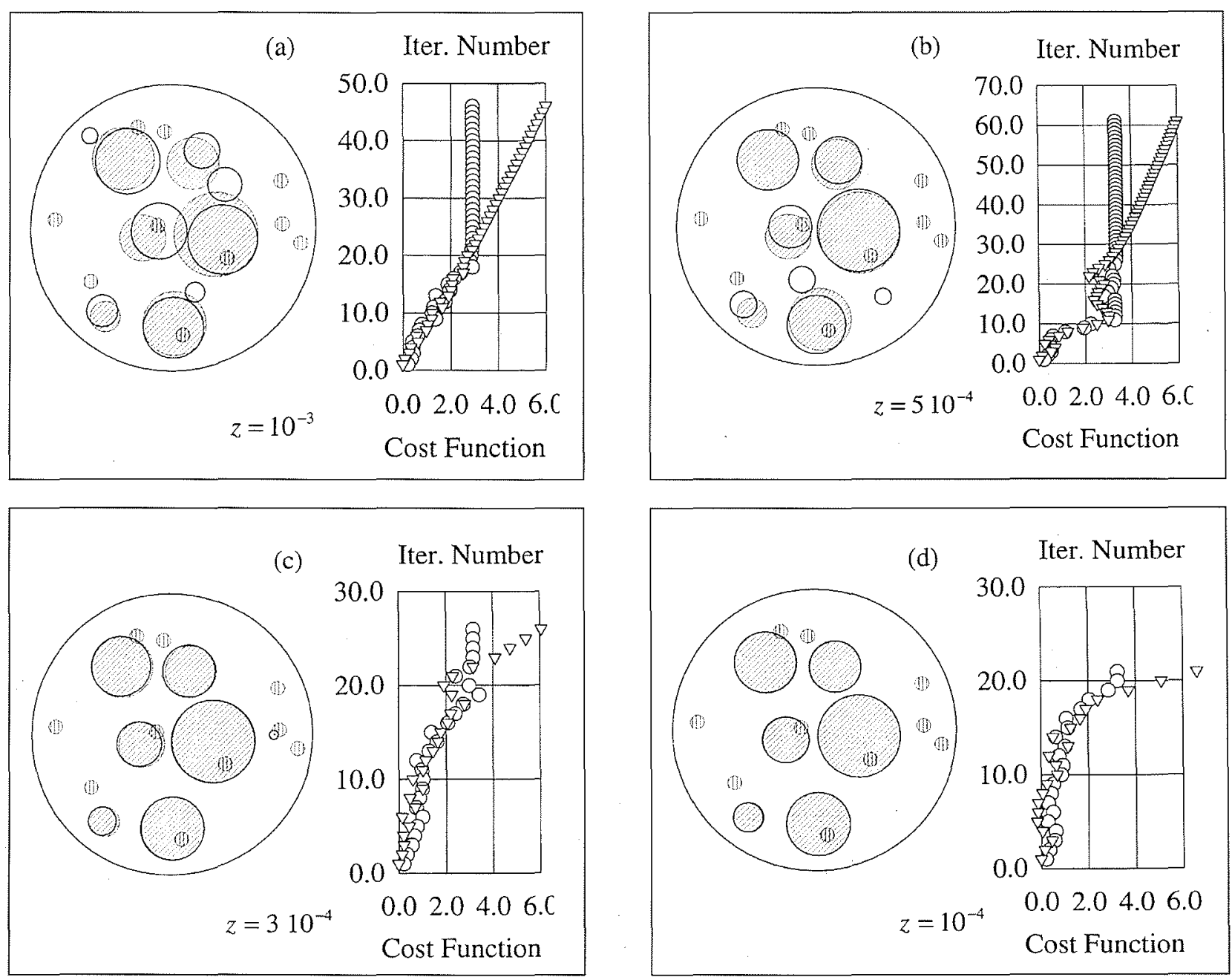

9. Reconstructions à partir de données simulées et prenant en compte les effets d'impédance d'interface. La reconstruction a lieu sans prise en compte de ces effets. Voir la légende de la figure 7 pour les définitions. L'importance de l'impédance d'interface $z$ diminue depuis $(a)$ jusqu'à $(d)$. La reconstruction est correcte pour les faibles valeurs de $z$ et surestime systématiquement le taux de présence moyen en créant des artefacts. La fonction objectif $(O)$ ne tend pas non plus dans ce cas vers zéro mais vers une valeur proportionnelle à l'impédance. 
Taille de l'inclusion (Rec. 2D)
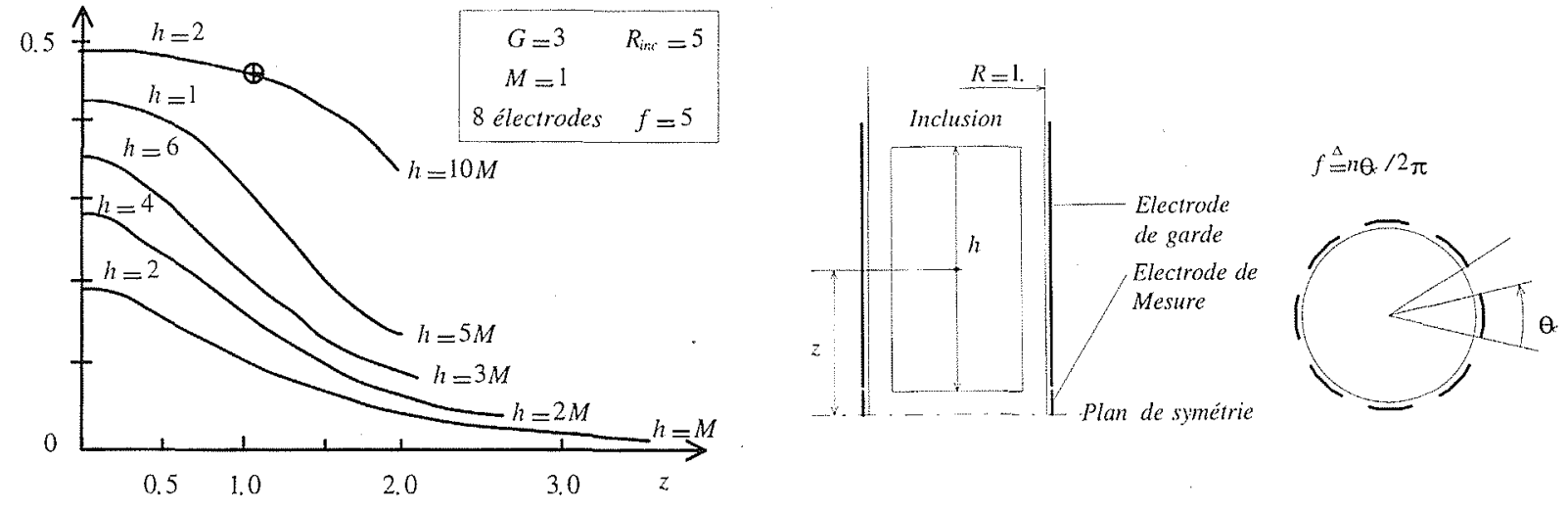

10. Influence des effets 3D sur la reconstruction tomographique. Les données sont simulées en 3D, la reconstruction est 2D. Le capteur contient une inclusion cylindrique de longueur $h$, de rayon $R_{i n c}$ et dont le centre de gravité est à une distance $z$ de l'axe de symétrie. Les électrodes de garde ont une longueur $G$, l'électrode de mesure, $2 \mathrm{M}$.

les effets d'impédance d'interface revient à surestimer l'impédance du milieu et l'algorithme a en conséquence tendance à surestimer le taux de présence. Il apparaît alors des inclusions fantômes ou bien les inclusions existantes sont dilatées ou rapprochées de la périphérie. Ces distorsions accordent, en effet, plus de poids aux inclusions.

Le problème du capteur plan, à taux de vide nul et à $n$ électrodes présente une invariance par rotation de $2 \pi / n$. Sur une maquette à 4 électrodes de $10 \mathrm{~cm}$ de diamètre et de $1 \mathrm{~cm}$ d'épaisseur, on a pu noter des défauts de symétrie d'environ $50 \Omega$ sur une mesure de $4,15 \mathrm{k} \Omega$. Ces défauts probablement dus à des états de surface d'électrode différents ou bien encore à des défauts géométriques ont des conséquences importantes sur la reconstruction car ils introduisent un biais systématique. Il est impératif de les compenser avant de tenter toute reconstruction. La seule procédure utilisable consiste à pratiquer un nombre suffisant d'expériences de référence et de recaler le modèle géométrique et électrique du capteur sur ces données. La position réelle des électrodes ainsi que la valeur exacte des impédances interfaciales étant déterminées par identification sur ces expériences de recalage, leurs effets seraient ensuite automatiquement compensés par la suite.

\section{DECONSTRUCTION DE DONNÉES RÉALISTES}

La MEF tridimensionnelle a été mise en œuvre, d'une part, pour optimiser la taille des électrodes de garde et de mesure (dans le sens de l'axe de la conduite) et, d'autre part, pour obtenir des données simulées représentatives d'un capteur réel.

Cette vérification n'a jamais été entreprise, à notre connaissance, et le plus souvent les algorithmes de reconstruction ne sont utilisés que sur des données simulées à l'aide de la méthode de calcul direct dont l'algorithme de reconstruction fait usage itérativement. C'est une procédure qui ne permet pas de juger pleinement de l'efficacité de l'algorithme de reconstruction 2D.
La figure 10 montre qu'une inclusion reconstruite avec un algorithme 2D est toujours plus petite qu'elle n'est en réalité même lorsqu'elle est centrée sur les électrodes de mesure. Il faut de plus que l'inclusion soit d'une longueur importante devant le rayon de la conduite et la longueur des électrodes de mesure pour obtenir une valeur correcte du rayon de l'inclusion reconstruite. De plus, lorsque celle-ci quitte le volume de mesure, mais en en demeurant au voisinage, la reconstruit bidimensionnelle fait apparaître une inclusion fantôme. $\mathrm{Ce}$ "fantôme " persiste d'autant plus longtemps que l'inclusion est longue.

Ces deux effets sont de plusieurs ordres de grandeurs supérieurs à tous ceux que l'on vient d'évoquer et semblent définitivement condamner l'utilisation de la tomographie d'impédance 2D pour la mesure précise du taux de vide en écoulement diphasique.

\section{CONCLUSIONS}

L'algorithme de reconstruction que l'on vient de présenter est particulièrement adapté aux problèmes diphasiques puisqu'il utilise toutes les informations connues sur le milieu comme la conductivité de chaque phase et la nature discontinue par morceau de la distribution de conductivité. De plus, en schématisant le milieu par un ensemble d'inclusions on optimise l'usage du peu d'informations indépendantes que le nombre limité d'électrode du milieu peut livrer.

Malgré ces précautions, et en dépit de l'excellent comportement numérique de l'algorithme de reconstruction vis-à-vis du bruit, le mauvais conditionnement du problème inverse de la conduction électrique se manifeste néanmoins: même si l'on possède un algorithme idéalement performant pour reconstruire des données très bruitées, la convergence a toujours lieu vers un minimum local de la fonction objectif dont la valeur ne dépend que du niveau de bruit. Il n'existe alors plus de critère objectif discriminant les reconstructions réussies, mais bruitées, de reconstructions erronées. 
De plus, les effets d'impédance d'interface introduisent des images fantômes que l'on ne peut éviter qu'à l'aide d'un recalage précis du modèle numérique du capteur. Enfin, lorsque l'on reconstruit des données simulées mais néanmoins représentatives de capteurs tridimensionnels on met également en évidence des artefacts de reconstruction très importants.

Pour toutes les raisons résumées ici, il semble imprudent de suggérer de développer plus avant la tomographie d'impédance en tant que technique de mesure précise du taux de présence local en écoulement diphasique pour toutes les configurations d'écoulement. La tomographie d'impédance à néanmoins un potentiel d'imagerie évident qui est bien démontré et pourrait être utilisée dans certaines configurations géométriques que les outils et les procédures présentés ici sont à même d'analyser.

\section{Remerciements}

Les auteurs remercient Monsieur Claude Favreau du CEA-Grenoble pour ses conseils et sa participation active dans les phases de définition du projet.

$L$ étude a été financée conjointement par le Commis. sariat a lénergie Atomique et le Groupement de Recherche du CNRS 1027 : Ecoulements diphasiques. Modélisation Multidimensionnelle et Techniques de Mesure 》.

\section{Références}

[1] JONES O.C., LIN J.T., OWACIK L. \& SHU L. (1993). Impedance imaging relative to gas-liquid systems, Nuclear Engineering And Design, n० 141, pp. 159-176.
[2] REINECKE N. \& MEwes D. (1995). - Visualization of transient two-phase flows using multielectrode capacitance sensors for electrical tomography, Proc. First Int. Symp. on Two-Phase Flow Modelling and Experimentation, G. P. Celata \& R. K. Shah Eds., Edizioni ETS, Pise, Italie, Vol. 2. pp. $739-750$.

[3] Peytraud J.F. (1995). - Etude de la tomographie électrique pour la mesure du taux de vide local en écoulements diphasiques. Doctorat de l'Institut National Polytechnique de Grenoble, France.

[4] ANDERSEN J.B, \& BERNTSEN (1988). - Quasi-static profile reconstruction of a circular cylinder, Joumal of Electromagnetic Waves and Applications, vol. 2, n5/6, pp. 429-444.

[5] Seagar A.D. \& Bates R.H.T. (1985). - Full-wave computed tomography, part 4 : low frequency electric current CT, IEE Proceeding Part A, vol. 132, pp. 455-459.

[6] Berntsen S., Andersen J.B. \& Gross E. (1991). - A general formulation of applied potential tomography, Radio Science, vol. $26, \mathrm{n}^{\circ} 2, \mathrm{pp} .535-540$.

17] Brebbia C.A. \& Dominguez J. (1992). - Boundary Elements, an Introductory Course, Computational Mechanics Publications, Southampton, UK.

[8] HESS J.L. \& SMITH A.M.O. (1967), - Calculation of potential about arbitrary bodies, Progress in aeronatical science, Kücheman, D., Ed., vol. 8, pp.1-138.

[9] Lemonnier H., Peytraud J.F. (1995). - A new algorithm for impedance imaging of two-phase flows, Proc. of the Forum on Measuremen Techniques in Mulniphase Flows, IMEC \& E, nov. 12-17, San Francisco, CA, USA.

[10] MarquadT D.W. (1963). - An algorithm for least-squares estimation of non linear parameters, SIAM Jom of Applied Mathematics, vol. 11, pp. 431-441. 